\title{
Resistive loading by a finite plasma cylinder
}

\section{By D. E. HASTI, $\uparrow$ M. E. OAKES AND H. SCHLÜTER}

Department of Physics, University of Texas at Austin, Austin, Texas

(Received 20 April 1970)

The loading resistance of a coil-plasma system operated near the lower hybrid resonance in hydrogen and argon has been measured. The results are consistent with the predictions of a theoretical model that includes finite plasma effects.

\section{Introduction}

Radio-frequency heating of plasmas has primarily concentrated on resonances at the cyclotron frequencies. The ion cyclotron resonance has received much attention because of its associated collisionless heating of ions. For waves propagating nearly perpendicular to a static magnetic field the resonant frequencies for high plasma density may approach the upper and lower hybrid frequencies. The lower hybrid resonance which lies between the electron and ion cyclotron resonances has until recently received little attention. Schlüter \& Ransom (1965) have studied this resonance in a mirror geometry using light gases. Brice \& Smith (1964) have suggested that noise signals received by the Alouette I satellite are associated with this resonance. Fredricks (1968), using the kinetic dispersion relation, studied this resonance for perpendicular propagation in an infinite homogeneous plasma. Stix (1965) has considered this resonance in an inhomogeneous plasma and has proposed a possible heating scheme using mode conversion.

Recently a theoretical treatment in a finite geometry was carried out by Skipping, Oakes \& Schlïter (1969). The radio-frequency impedance of a coil concentric with a homogeneous magnetized plasma cylinder of finite length determined by conducting end plates was computed and studied near the lower hybrid resonance and also near coupling resonances. The cold plasma dispersion relation was used and collisions were included. The conditions of this linear theoretical model have been approximated in an experiment and the results are reported. The perturbations in the experiment are large and a more complete solution would require a nonlinear model.

In the following section a brief outline of the theoretical calculation is given, for details the reader is referred to the original paper.

† Now at Austin Research Associates, Austin, Texas.

$\ddagger$ Now at Ruhr-Universität Bochum, Bochum, West Germany. 


\section{Theory}

The model of the plasma-coil system is a uniform plasma cylinder of radius $p$ and length $L$ bounded by conducting end walls infinite in the radial direction with the oscillator coupling coil approximated by an azimuthal current sheet of length $a$ and radius $s(s>p)$ concentric with the plasma cylinder. The plasma is immersed in a uniform axial magnetic field and is described by fluid equations for the electrons, ions and neutrals in which pressure, viscous and gravitational terms are neglected but collisions are retained. The equations are linearized, the time dependence $e^{-i \omega t}$ is assumed and quasi-neutrality is used. The fluid equations are solved with Maxwell's equations for three regions: (I) in the plasma, (II) in the vacuum between the coil and the plasma, (III) in the vacuum outside the coil. Using the appropriate boundary conditions at the inter-faces between the three regions and the radiation condition for the far vacuum region, the Fourier transformed electric field components are determined.

In the plasma

$$
\begin{gathered}
e_{r}=A_{n}^{(1)} J_{1}\left(\eta_{\perp 1} k_{0} r\right)+A_{n}^{(2)} J_{1}\left(\eta_{\perp 2} k_{0} r\right), \\
e_{\theta}=\frac{K_{r \theta} A_{n}^{(1)}}{K_{r r}-\eta_{1}^{2}} J_{1}\left(\eta_{\perp 1} k_{0} r\right)+\frac{K_{r \theta} A_{n}^{(2)}}{K_{r r}-\eta_{2}^{2}} J_{1}\left(\eta_{\perp 2} k_{0} r\right), \\
e_{z}=\frac{\eta_{\|} \eta_{\perp 1} A_{n 1}^{(1)}}{K_{z z}-\eta_{\perp 1}^{2}} J_{0}\left(\eta_{\perp 1} k_{0} r\right)+\frac{\eta_{1} \eta_{\perp 2} A_{n}^{(2)}}{K_{z z}-\eta_{\perp 2}} J_{0}\left(\eta_{\perp 2} k_{0} r\right),
\end{gathered}
$$

where the labels (1) and (2) denote the two values of $\eta_{\perp}$ determined from the dispersion relation

$$
\left(K_{z z}-\eta_{\perp}^{2}\right)\left(K_{r r}^{2}+K_{r \theta}^{2}-\eta^{2} K_{r r}\right)-\eta_{\|} K_{z z}\left(K_{r r}-\eta^{2}\right)=0
$$

and $\eta=\eta_{\perp}+\eta_{\|}$. The elements of the dielectric tensor are:

$$
\begin{aligned}
& K_{r r}=K_{\theta \theta}=1+\frac{\omega_{p}^{2} Q}{Q^{2}-\omega^{2} \omega_{e}^{2}}, \\
& K_{r \theta}=-K_{\theta r}=\frac{i \omega \omega_{e} \omega_{p}^{2}}{Q^{2}-\omega^{2} \omega_{e}^{2}}, \\
& K_{z z}=1-\frac{\omega_{p}^{2}}{\omega(\omega+i \gamma)},
\end{aligned}
$$

where

$$
\omega_{e}=\frac{e B_{0}}{m c}, \quad \omega_{i}=\frac{e B_{0}}{M c}, \quad \omega_{0}^{2}=\omega_{e} \omega_{i}, \quad Q=\omega_{0}^{2}-\omega^{2}-i \gamma \omega,
$$

and $\gamma$ is the collision frequency.

In the vacuum regions $K_{r r}=K_{z z}=1$ and $K_{r \theta}=0$ leading to solutions.

Region $p \leqslant r \leqslant s$ :

$$
\begin{aligned}
& e_{r}=\eta_{\|} \alpha^{-1}\left[B_{n} I_{1}\left(\alpha k_{0} r\right)-C_{n} K_{1}\left(\alpha k_{0} r\right)\right] \\
& e_{\theta}=F_{n} I_{1}\left(\alpha k_{0} r\right)+G_{n} K_{1}\left(\alpha k_{0} r\right) \\
& e_{z}=B_{n} I_{0}\left(\alpha k_{0} r\right)+C_{n} K_{0}\left(\alpha k_{0} r\right) .
\end{aligned}
$$


Region $r \leqslant s$ :

$$
\begin{aligned}
& e_{r}=-\eta_{11} \alpha^{-1} D_{n} K_{1}\left(\alpha k_{0} r\right), \\
& e_{\theta}=H_{n} K_{1}\left(\alpha k_{0} r\right), \\
& e_{z}=D_{n} K_{0}\left(\alpha k_{0} r\right) .
\end{aligned}
$$

In equations (8)-(13) $\alpha^{2} \equiv\left(\eta_{0}^{2}-1\right)$ and $I_{j}\left(\alpha k_{0} r\right)$ and $K_{j}\left(\alpha k_{0} r\right)$ are modified Bessel function of the first and second kinds. The coefficients in the expressions for the field components are functions of the coil and plasma radius, coil and plasma length, and indices of refraction of the plasma.

The plasma loading resistance is calculated by assuming no ohmic losses in the coil and integrating the Poynting vector over a surface which completely encloses the coil, this leads to the expression

$$
P=\frac{1}{2} \operatorname{Re}\left[-I^{*} \frac{2 \pi s}{a} \int_{\frac{t}{2}(L-a)}^{\frac{1}{2}(L+a)} E_{\theta}(s, z) d z\right]
$$

for the total power input to the coil. The $I$ in equation (14) is the current in the coil which appears when the current per unit length,

$$
\begin{aligned}
& J=I / a \text { for } \frac{1}{2}(L-a) \leqslant z \leqslant \frac{1}{2}(L+a), \\
& J=0 \text { elsewhere, }
\end{aligned}
$$

is used in the expression for the discontinuity in $B_{z}$ at the coil. The factor multiplying $I^{*}$ in equation (14) is the peak coil voltage $V$. Using the Ohm's law relation for $a c$ circuits the current in equation (14) can be eliminated, and taking the coil-plasma system to behave as a parallel equivalent circuit allows the power to the coil to be expressed as

$$
P=\frac{|V|^{2}}{2 R}
$$

where $R$ is the resistive part of the impedance of the equivalent circuit. If the power loss from radiation by the coil is neglected, i.e. the plasma is assumed to absorb all the power input to the coil, $R$ in equation (16) can be identified as the plasma loading resistance. The conducting end walls lead to the condition that outgoing waves are cut off for $k_{\mathbb{1}} / k_{0}>1$. The power can be calculated by integrating the Poynting vector either over a surface completely enclosing the coil, or over a surface enclosing the plasma. Using the electric fields found above and $V$ evaluated from equation (14), one finds the following expression for the plasma loading resistance $R$ :

$$
R(\mathrm{ohms})=\frac{1.92 \times 10^{3} k_{0} L\left(\frac{s}{a}\right)^{2}\left|\sum_{n}^{\prime} \frac{K_{1}^{2}(\alpha S)}{n^{2}} \sin ^{2}\left(\frac{n \pi a}{2 L}\right)\left(\frac{H_{n}}{F_{n}}\right)\right|^{2}}{\sum_{n}^{\prime} \frac{K_{1}^{2}(\alpha S)}{n^{2}} \sin ^{2}\left(\frac{n \pi a}{2 L}\right) \operatorname{Im}\left(\frac{H_{n}}{F_{n}}\right)} .
$$

The prime on the summation indicates the sum is to be carried out over odd integers. This expression is numerically evaluated using the geometry of the experimental machine, i.e. the plasma radius $p(3 \mathrm{~cm})$, the coil radius $s$ $(4.75 \mathrm{~cm})$, the coil length $a(3.8 \mathrm{~cm})$ and the separation between the conducting 
end walls $L(5 \mathrm{l} \mathrm{cm})$. Collision frequencies and electron densities determined from experimental measurements are used as input to the program for each value of $B_{0}$ giving curves of $R$ vs. $B_{0}$ (or $\omega_{0} / \omega$ ).

An expression for the loading resistance for axisymmetric oscillations with no $\theta$ dependence has been obtained by Körper (1960) by assuming an infinite length plasma and coil, calculating the loading resistance per unit length. The total resistance for a coil of length $a$ is (in ohms)

$$
R=\frac{7 \cdot 2 \times 10^{12} \pi^{2} p^{2} \omega}{a c^{2}} \frac{\left|1+\frac{1}{2}\left[\left(\frac{s}{p}\right)^{2}-1\right]\left(k_{0} p \eta\right) \frac{J_{0}\left(k_{0} p \eta\right)}{J_{1}\left(k_{0} p \eta\right)}\right|^{2}}{\operatorname{Im}\left[\left(k_{0} p \eta\right) \frac{J_{0}\left(k_{0} p \eta\right)}{J_{1}\left(k_{0} p \eta\right)}\right]},
$$

where $\eta$ for the extraordinary wave is determined from equation (4) with $\eta_{\sharp}$ set to zero.

\section{Experiment}

A steady-state r.f. discharge was produced by inductive coupling of a modified Colpitts oscillator through a single turn coil around the discharge tube while the gas pressure was regulated at a predetermined value. The magnetio field was

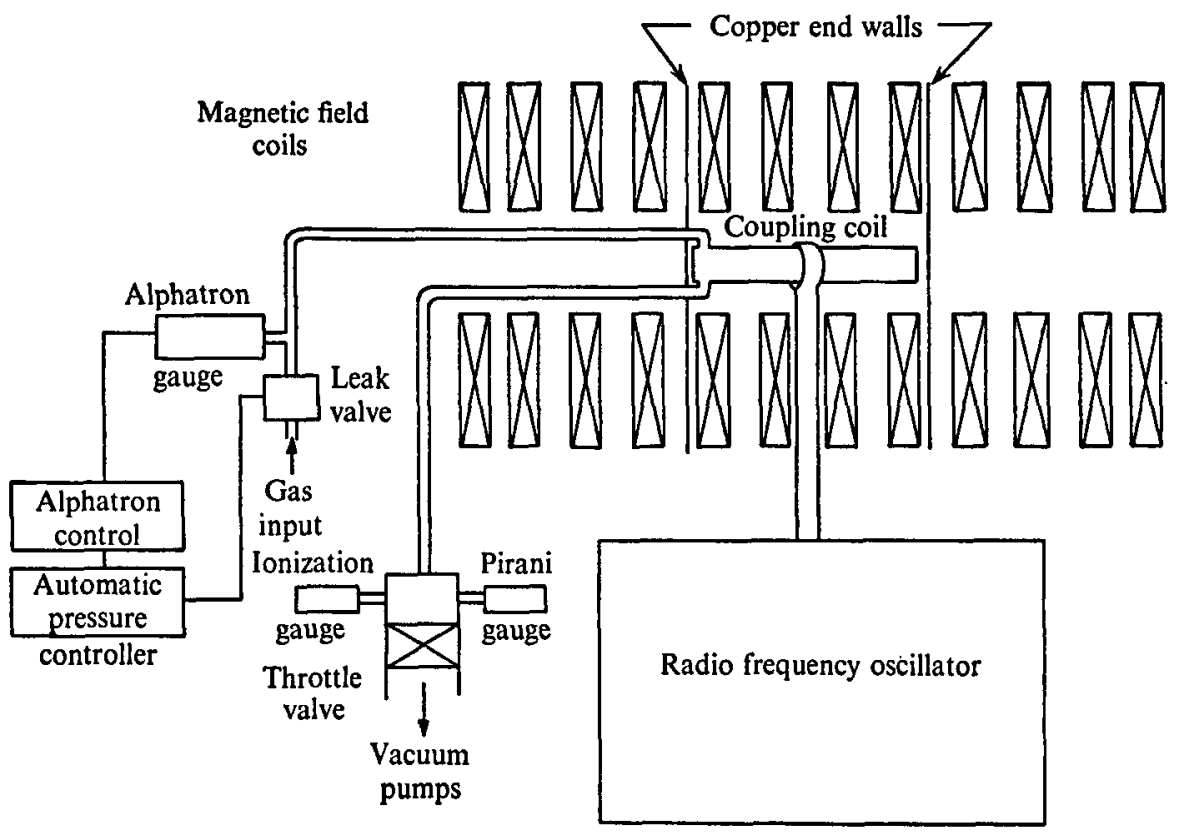

FIGURE 1. Schematic of the apparatus.

then swept while simultaneous plots were made of the r.f. grid voltage and power transfer to the plasma. The coil voltage is determined from the grid voltage so these two measurements determine experimentally the plasma loading resistance by using equation (16). A schematic of the system is shown in figure 1 .

A maximum of $10 \mathrm{~kW}$ power could be applied to the oscillator tube but to insure stable operation over the range of loading conditions encountered in a 
sweep of the magnetic field, typical power levels on the order of $3 \mathrm{~kW}$ at $24 \cdot 4 \mathrm{MHz}$ in the hydrogen experiments and $4.5 \mathrm{~kW}$ at $25.5 \mathrm{MHz}$ in the argon experiments were used. The different frequencies and power levels in the two oases resulted from changes in the feedback capacitance and bias resistance necessary to maximize power transfer while maintaining the oscillator in a stable mode over the sweep of the magnetic field. A careful analysis of the frequency spectrum of the oscillator was made to determine that the output was essentially c.w. for all magnetic fields and pressures before the experiments were conducted in each gas; periodic checks confirmed continuing proper operation. Some small variation in frequency over the full range of magnetic field was observed due to changes in the oscillator loading but in all cases this variation was less than $1 \%$.

The discharge vessel was a quartz cylinder $47 \mathrm{~cm}$ long and $6 \mathrm{~cm}$ in diameter surrounded by a water jacket extending to within $5 \mathrm{~cm}$ of the ends. The water flow was regulated and metered and thermo-couples in the input and output allowed calorimetric power measurements to determine the power absorbed by the plasma. Previous measurements on similar hydrogen discharges using water caps on the ends of the discharge vessel and an opaque coolant have shown that the power radiated by the plasma and the losses through the ends of the discharge vessel not covered by the water jacket are negligible (Schlüter \& Ransom, 1965). Tests without plasma have shown virtually zero power transfer to the cooling system of the vessel. The theoretical loading resistances to be compared to in this investigation do not include (experimentally undetermined) power losses to the outside region of the coil. Ohmic losses in the coupling coil are neglected in the calculations. For weak loading of the oscillator, this may lead to errors in the comparison of experiment and theory (see remarks below on saturation effects of the oscillator).

The gas pressure was regulated with a Granville-Phillips Automatic Pressure Controller using an Alphatron gauge for input. This system allowed the pressure to be maintained to within $\pm 5 \%$ in the range of the experiments, $0.025-0.262$ Torr; a base pressure of about $3 \times 10^{-7}$ Torr was maintained throughout the period the experiments were being conducted. The pressure controlling system and diagnostic instruments were carefully shielded against r.f. and magnetic perturbations.

The magnetic field was produced with a solenoid $140 \mathrm{~cm}$ long with a $22 \mathrm{~cm}$ bore made up of 12 coils, each $6.5 \mathrm{~cm}$ wide, spaced to give a length of homogeneous field of $1 \mathrm{~m}\left( \pm 1 \%\right.$ variation of $B_{0 z}$ on the axis) and $15 \mathrm{~cm}$ across the bore of less than $1 \%$ variation in $B_{0 z}$. The magnetic field was varied with a motor driven variable power supply which allowed the slow sweep speeds necessary to maintain thermal equilibrium for the calorimetric measurements as the field was swept. The range of fields in continuous sweep, measured with a Hall effect gaussmeter, was $550-3600 \mathrm{G}$ for the argon experiments and 100 to $2000 \mathrm{G}$ for the hydrogen experiments.

Typical grid voltage and power absorption curves are shown in figure 2 for hydrogen and in figure 3 for argon. The curves are obtained from two $x-y$ recorders with the r.f. grid voltage as the $y$ input of one, the e.m.f. developed between the water jacket thermocouples as the $y$ input of the other, and the solenoid current 
as the $x$ input for both. The experimental loading resistance curves were determined by taking grid voltage drop and power absorbed values from three or four curves like these for each pressure. The coil voltage is proportional to the grid voltage. The experimental loading resistance curves are plotted in relative units.
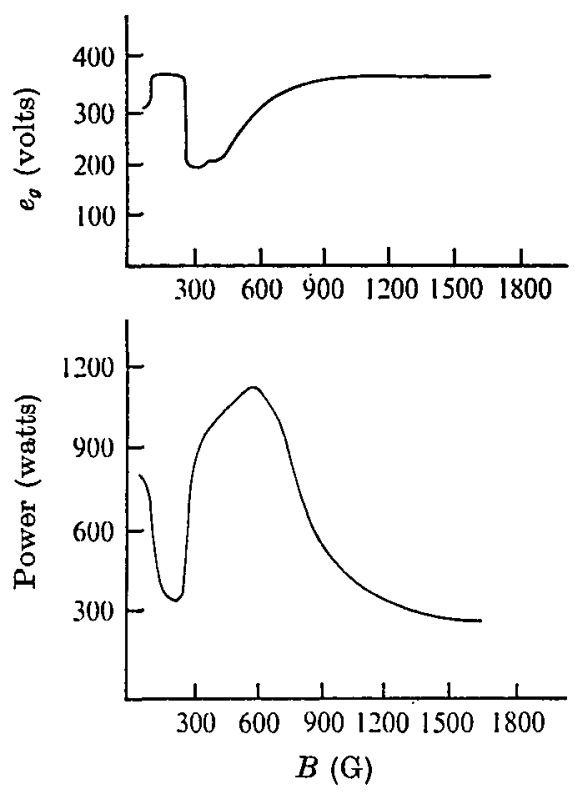

Figure 2. Typical r.f. grid voltage and power curves for hydrogen. (0.025 Torr).

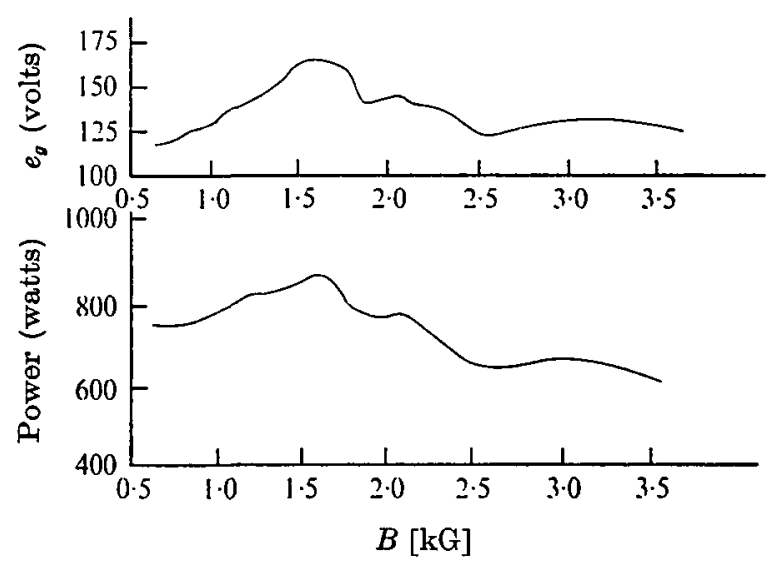

FrquRe 3. Typical r.f. grid voltage and power curves for argon (0.048 Torr).

The electron density was measured with a $4 \mathrm{~mm}$ microwave interferometer propagating across the discharge using the ordinary wave. The electron density as a function of magnetic field seen by the oscillator was put into the numerical calculations of loading resistance. The experimentally measured average values of $N_{e}$ were reduced by a factor of 0.5 for all hydrogen data and by 0.732 for the 
0.048 Torr, 0.660 for the 0.148 Torr, and 0.533 for the 0.262 Torr argon cases. The reduced values are shown in figures 4 and 5 . The factors were chosen to give the best fit of the positions of the extrema in the loading resistance curves over the range of magnetic field where $N_{e}$ is approximately constant; in argon the different factors reflect a steepening of the density gradient with increasing pressure as determined from measurements of $N_{e}$ across the axis and $1.5 \mathrm{~cm}$ above the axis of the discharge tube. Parameters used to identify theoretical curves refer to the experimental electron densities and the neutral number densities used in calculating collision frequencies. To calculate collision frequencies to be put into numerical computations of loading resistance, electron and neutral temperatures from previous measurements on similar machines were used $\left(T_{e} \sim 10 \mathrm{eV}, T_{N} \sim 0.2 \mathrm{eV}\right.$ ) (Schlüter \& Ransom, 1965; Schlüter \& Avila, 1966).

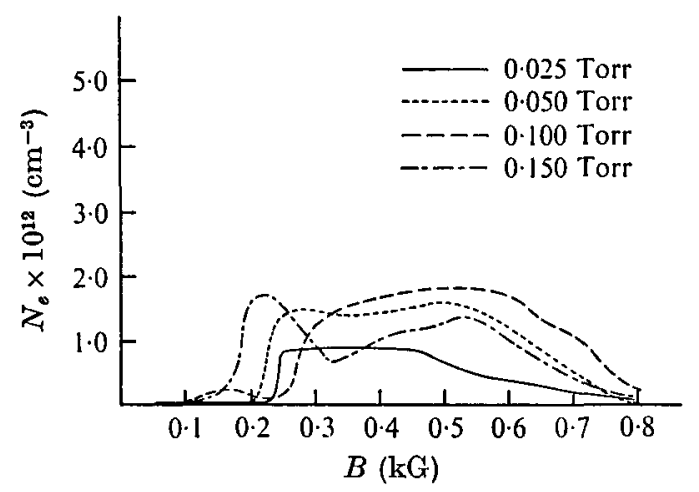

FraURe 4. Reduced electron densities vs. $B$ in hydrogen.

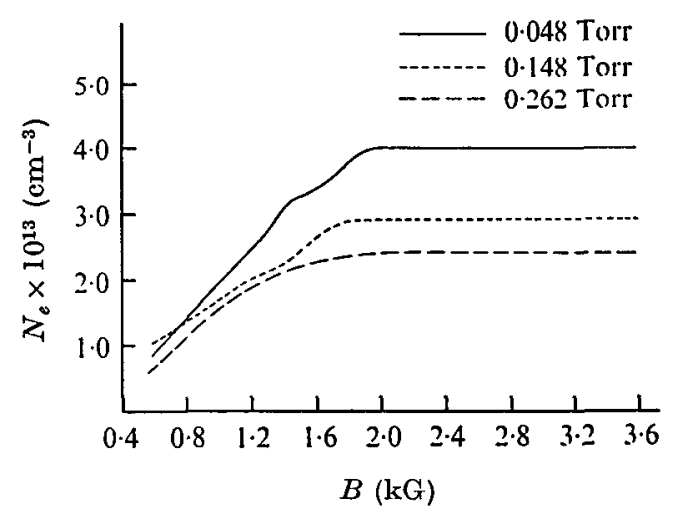

Frgure 5. Reduced electron densities vs. $B$ in argon.

\section{Results and discussion}

Plots of loading resistance $v s . \omega_{0} / \omega$ from experimental measurements and numerical computations using both equations (17) and (18) with experimentally determined parameters are shown in this section. The collision frequencies used in the theory are a sum of $\gamma_{e i}$ and $\gamma_{e n}\left(\gamma_{i n} \gg \gamma_{e i}, \gamma_{e n}\right)$ with $\gamma_{e i}$ obtained from 
Spitzer (1962) and $\gamma_{e n}$ from Brown (1959). To compute the neutral density in the discharge tube it is assumed that the pressure remains constant (at the controlled value) when the discharge is turned on while the number density of neutrals is reduced because of the increased gas temperature: the effect of thermal transpiration causes no drastic uncertainty of this reduction. The essential effect of increased collision frequency on the theoretical curves is to decrease the difference in magnitude between maxima and minima while not changing the $\omega_{0} / \omega$ positions or the structure of the loading resistance curves.

In figures 6-9 the experimental and theoretical loading resistance curves, plotted against $\omega_{0} / \omega$, are shown for the experiments in hydrogen; the different data points are for different runs. With increasing gas pressure, thus increasing collision frequenoy, the resonance curves become broader, as to be expected; there are, however, differences between theory and experiment. The most obvious discrepancies between theoretical and experimental curves occur where the slope of the theoretical curves is rather steep (in particular always for $\left.\omega_{0} / \omega \simeq 1 \cdot 5-2 \cdot 0\right)$. The experimental loading resistance is unable to follow the rapid rise in the theoretical curves because the oscillator has become saturated, i.e. the grid voltage has increased approximately to the value when no plasma is present. Therefore the reduced steepness of the experimental curves at high plasma loading resistances to a large extent stem from this saturation effect which is unavoidable when stable operation of the oscillator over a large range of loading is required (as in hydrogen, but not the argon case). Of course, the presence of density gradients, not explicitly accounted for in the theory, tends to smooth out additionally the loading curves in both the hydrogen and argon case. Possible overestimates in collision frequencies are expected to contribute only to a minor degree.

The situation in argon was quite different in that the range of loading resistance was much smaller and the oscillator could be set to load strongly into the plasma over the entire range of magnetic field (it should be pointed out that the oscillator could be operated at no more than a few hundred watts input with no plasma when in the configuration used for the argon experiments without drawing excessive grid current). In this case the relatively small variations of loading resistance predicted theoretically could be seen in the experimental curves as is shown in figures 10-12. As mentioned earlier, previous experiments in this type of discharge had shown that the power transferred to the ends of the discharge tube was negligible, however, the maximum magnetic field available in the previous experiments was less than half the maximum field in these argon experiments and it might be expected that end losses would become more important at the higher magnetic fields. Experimental difficulties in installing water jackets on the ends, while retaining the configuration of conducting end walls, dictated that the assumption of negligible losses to the ends be used for these relative measurements even at the higher magnetic fields of the argon experiments, however, the general trend of decreasing power with increasing magnetic field (and consequent increasing $R$ with increasing $\omega_{0} / \omega$ ) seen in the argon results might be attributed to some extent to power losses at the ends of the discharge tube. 


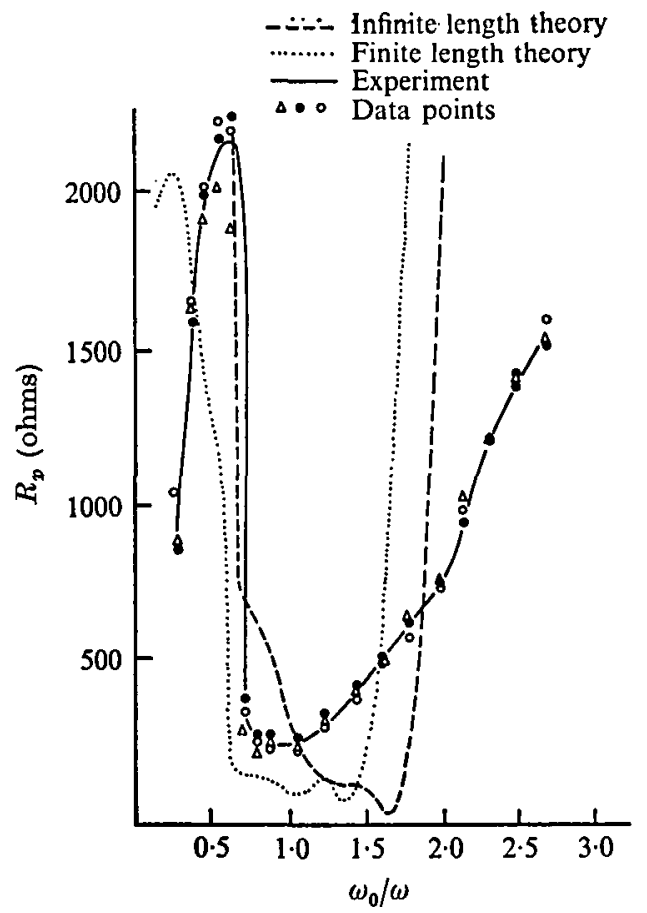

FiguRe 6. Experimental and theoretical $R_{p}$ vs. $\omega_{0} / \omega$ for 0.025 Torr in hydrogen. Experimental values are in relative units. The different data symbols indicate separate runs. $p=3 \mathrm{~cm}, s=4.75 \mathrm{~cm}, a=3.8 \mathrm{~cm}$ and $L=51 \mathrm{~cm}$.

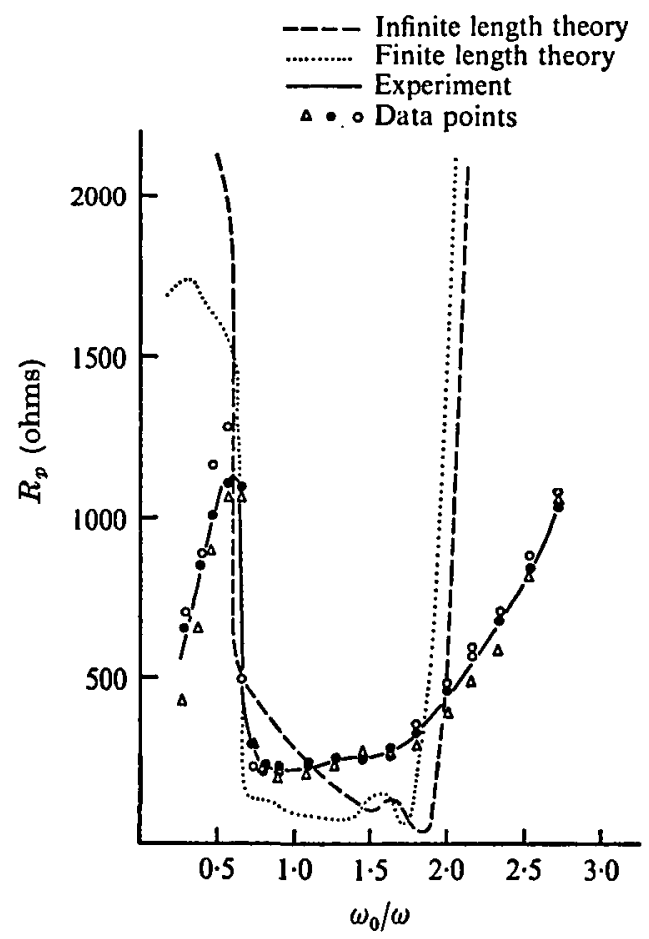

Fraure 7. Experimental and theoretical $R_{p} v s . \omega_{0} / \omega$ for 0.050 Torr in hydrogen. Experimental values in relative units, $p=3 \mathrm{~cm}, s=4.75 \mathrm{~cm}, a=3.8 \mathrm{~cm}$ and $L=51 \mathrm{~cm}$. 


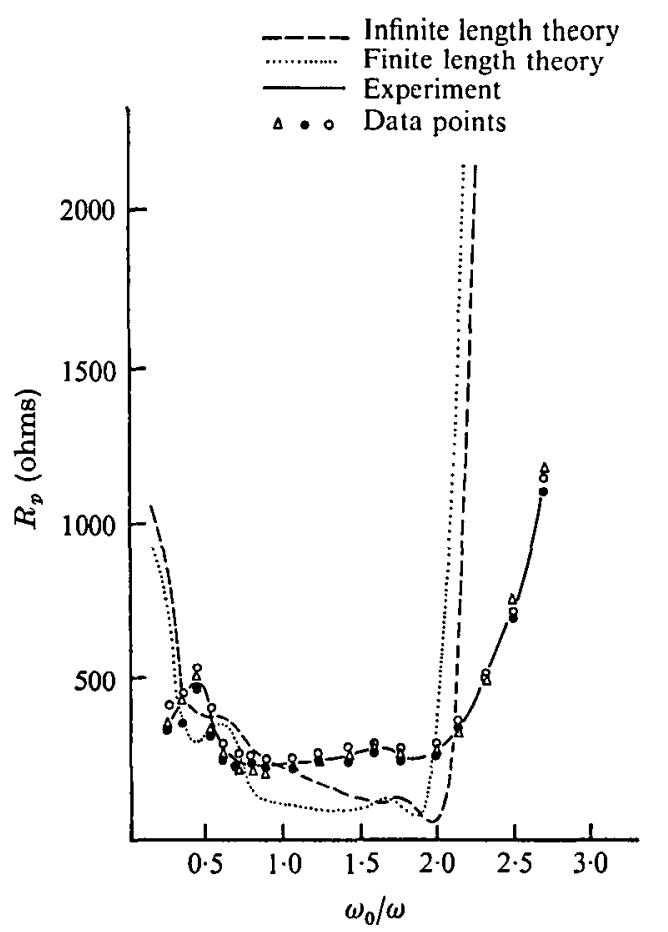

FigURE 8. Experimental and theoretical $R_{p} v s . \omega_{0} / \omega$ for $0 \cdot 100$ Torr in hydrogen. Experimental values in relative units. $p=3 \mathrm{~cm}, s=4.75 \mathrm{~cm}, a=3.8 \mathrm{~cm}$ and $L=51 \mathrm{~cm}$.

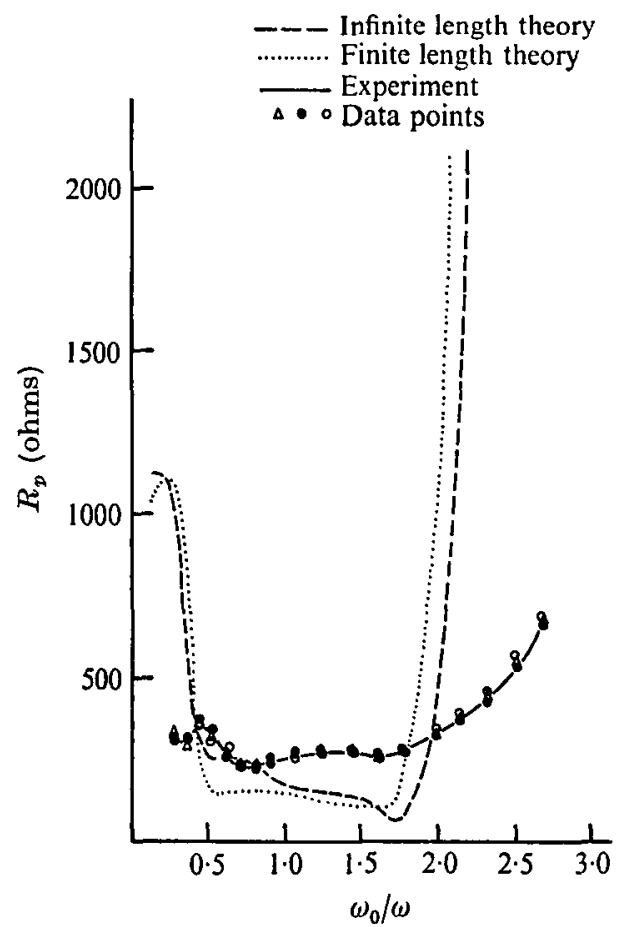

Frgure 9. Experimental and theoretical $R_{p} v s . \omega_{0} / \omega$ for 0.150 Torr in hydrogen. Experimental values in relative units. $p=3 \mathrm{~cm}, s=4.75 \mathrm{~cm}, a=3.8 \mathrm{~cm}$ and $L=51 \mathrm{~cm}$. 


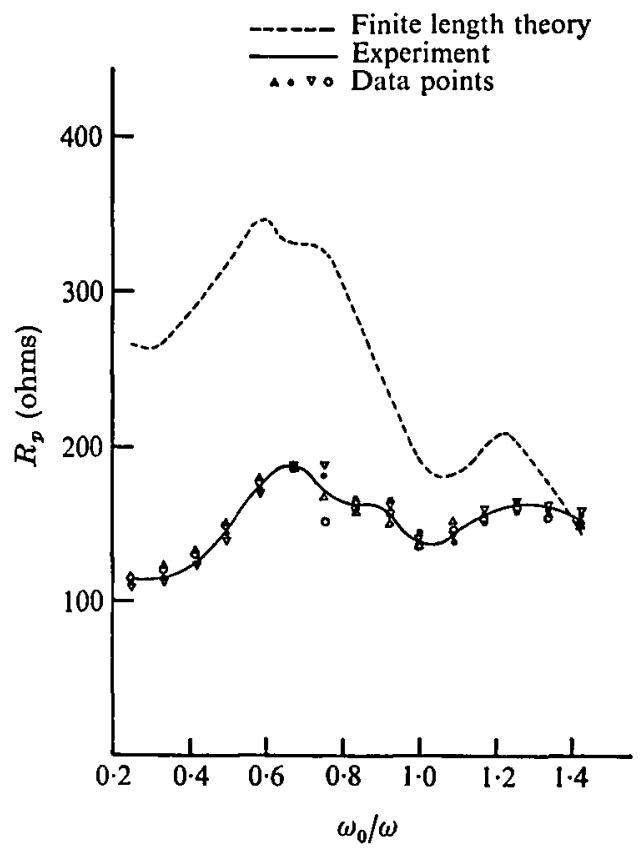

FIGURE 10. Experimental and theoretical $R_{p} v s . \omega_{0} / \omega$ for 0.048 in argon. Experimental values in relative units. $p=3 \mathrm{~cm}, s=4.75 \mathrm{~cm}, a=3.8 \mathrm{~cm}$ and $L=51 \mathrm{~cm}$.

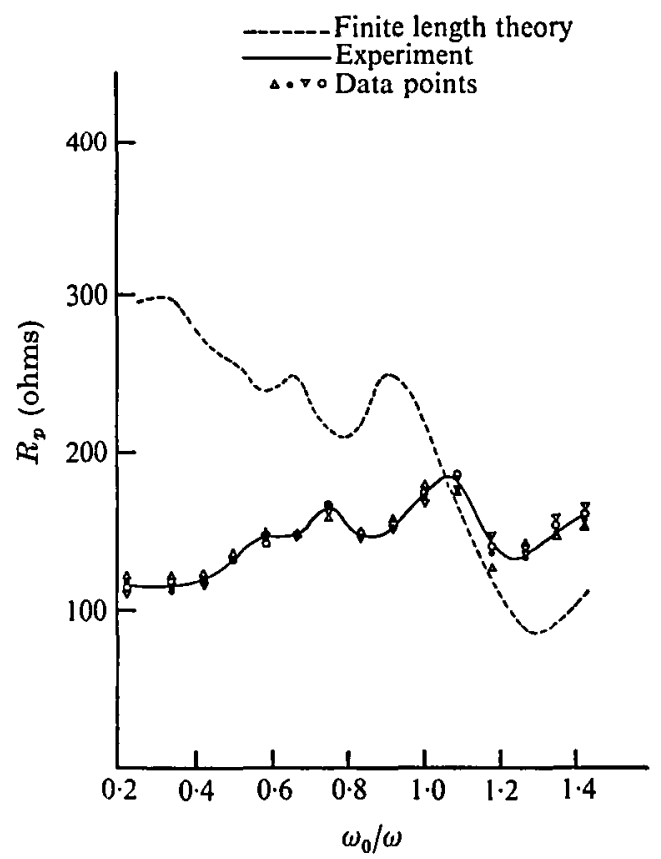

Frafre 11. Experimental and theoretical $R_{p} v s . \omega_{0} / \omega$ for 0.148 Torr in argon. Experimental values in relative units. $p=3 \mathrm{~cm}, s=4.75 \mathrm{~cm}, a=3.8 \mathrm{~cm}$ and $L=51 \mathrm{~cm}$. 




FraURe 12. Experimental and theoretical $R_{p} v s . \omega_{0} / \omega$ for 0.262 Torr in argon. Experimental values in relative units. $p=3 \mathrm{~cm}, s=4.75 \mathrm{~cm}, a=3.8 \mathrm{~cm}$ and $L=51 \mathrm{~cm}$.

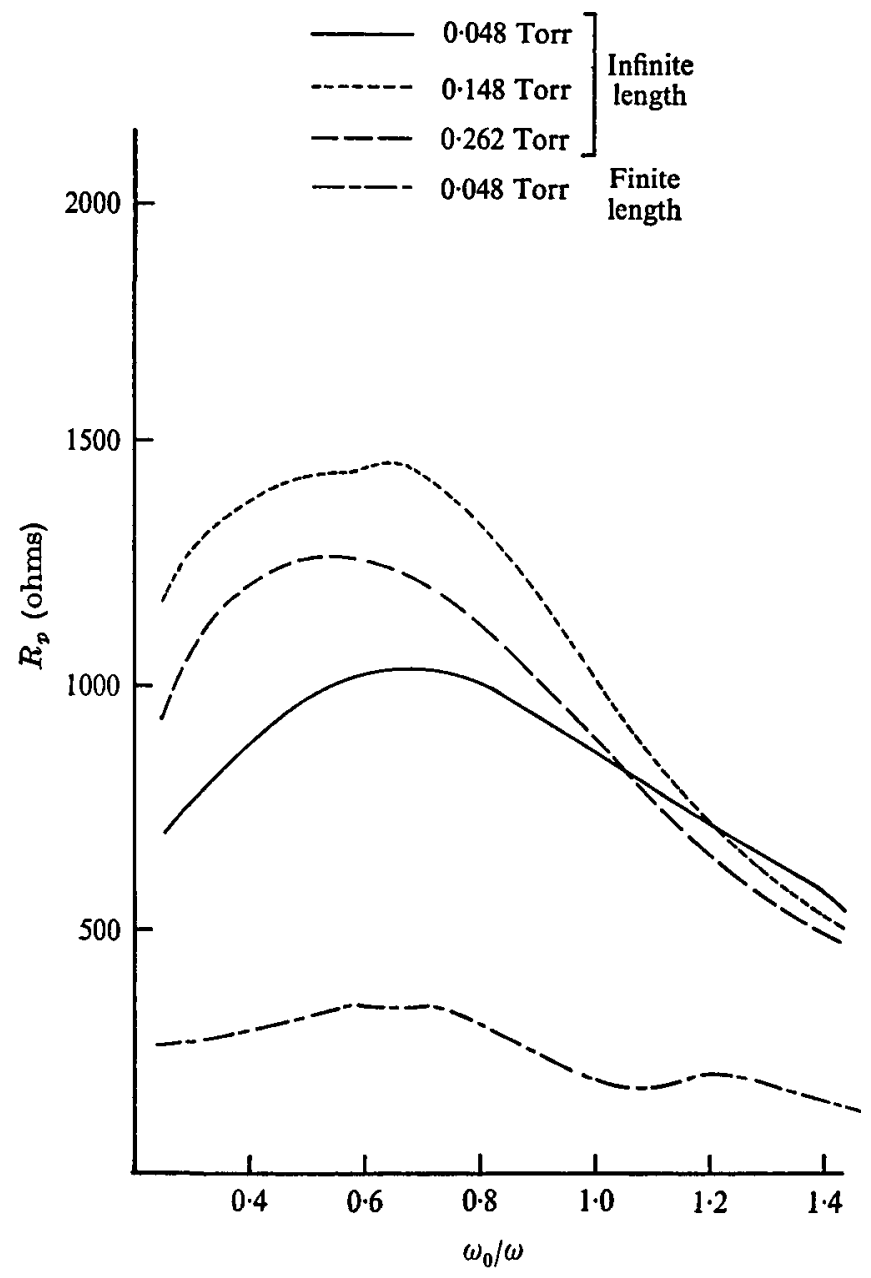

Figure 13. $R_{p} v s . \omega_{0} / \omega$ in argon (theory). $p=3 \mathrm{~cm}, s=4.75 \mathrm{~cm}, a=3.8 \mathrm{~cm}$ and $L=51 \mathrm{~cm}$ (finite length case). 
Figure 13 shows $R$ vs. $\omega_{0} / \omega$ from equation (18) (infinite length theory) for the argon data which can not be included on figures 10-12 because of the difference in magnitude of $R$. The infinite length case in argon exhibits almost none of the structure seen in the finite plasma calculation, whereas in hydrogen the two results are quite similar. This is due to an enhancement of the $k_{\|}$contributions for large ion masses. The dispersion relation for small $k_{\|}$approximates the perpendicular propagation case provided the ion mass is small; for heavier ions (such as argon) the refractive indices are strongly modified (Mills, Oakes \& Schlüter 1966).

The importance of higher Fourier components is apparent in the argon case. The loading resistance using only the lowest component $(\pi / L)$ yields a relatively smooth curve, however including $3 \pi / L$ introduces structure seen in the experiment; these results are characteristic of all the argon data.

\section{Summary}

The loading of a radio-frequency ring discharge has been studied at frequenoies near the lower hybrid resonance. The results are found to be described rather well by a recent linear calculation including finite plasma length, although density gradients are only accounted for by the use of averaged values in the calculations and large electric fields are present in the experiment. Structure observed in argon indicates the importance of higher axial Fourier components for heavy gases.

The authors wish to thank Mr Dillon H. McDaniel for his assistance with the experiments.

This work was supported by the National Science Foundation and the Texas Atomic Energy Research Foundation.

\section{REFERENCES}

Brice, N. M. \& Smith, R. L. 1965 J. Geophys. Res. 70, 71.

Brown, S. C. 1959 Basic Data of Plasma Physics. John Wiley.

Fredricks, R. W. 1968 J. Plasma Phys. 2, 197, 365.

KöRPER, K. 1960 Z. Naturforsch, 15 A, 220, 226, 235.

Mulls, G. S., OAKes, M. E. \& Schü̈TER, H. 1966 Phys. Lett. $21,45$.

SchlÜter, H. \& Ransom, C. J. 1965 Ann. Phys. (N.Y.) 33, 360.

SchLÜTER, H. \& AviLA, C. 1966 Astrophys. J. 144, 785.

SkIPping, C. R., OAkes, M. E. \& Schlüner, H. 1969 Phys. Fluids (to be published). SpItzer, L., Jr. 1962 Physics of Fully Ionized Gases. Interscience Publishers, Inc. Strx, T. H. 1965 Phys. Rev. Lett. 15, 878. 